\title{
Treatment planning comparison of volumetric modulated arc therapy with the trilogy and the Halcyon for bilateral breast cancer
}

\author{
Tao Sun ${ }^{\dagger}$, Xiutong Lin ${ }^{\dagger}$, Guifang Zhang, Qingtao Qiu, Chengqiang Li and Yong Yin ${ }^{*}$
}

\begin{abstract}
Background: The Halcyon is a new machine from the Varian company. The purpose of this study was to evaluate the dosimetry of the Halcyon in treatment of bilateral breast cancer with volumetric modulated arc therapy.

Methods: On CT images of 10 patients with bilateral breast cancer, four Halcyon plans with different setup fields were generated, and dosimetric comparisons using Bonferroni's multiple comparisons test were conducted among the four plans. Whole and partial arc plans on the Trilogy and the Halcyon, referred to as T-4arc, T-8arc, H-4arc and $\mathrm{H}$-8arc, were designed. The prescription dose was $50 \mathrm{~Gy}$ in 2-Gy fractions. All plans were designed with the Eclipse version 15.5 treatment planning system. The dosimetric differences between whole and partial arc plans in the same accelerator were compared using the Mann-Whitney $U$ test. The better Halcyon plan was selected for the further dosimetric comparison of the plan quality and delivery efficiency between the Trilogy and the Halcyon.
\end{abstract}

Results: Halcyon plans with high-quality megavoltage cone beam $C T$ setup fields increased the $D_{\text {mean, }} D_{2}$ and $V_{107}$ of the planning target volume (PTV) and the $V_{5}$ and $D_{\text {mean }}$ of the heart, left ventricle (LV) and lungs compared with other Halcyon setup plans. The mean dose and low dose volume of the heart, lungs and liver were significantly decreased in T-8arc plans compared to T-4arc plans. In terms of the $V_{5}, V_{20}, V_{30}, V_{40}$ and $D_{\text {mean }}$ of the heart, the $V_{20}, V_{30}, V_{40}$ and $D_{\text {mean }}$ of the $L V$, the $V_{30}, V_{40}, D_{\text {max }}$ and $D_{\text {mean }}$ of the left anterior descending artery $(L A D)$, and the $V_{5}$ and $V_{40}$ of lungs, $\mathrm{H}$-8arc was significantly higher than $\mathrm{H}$-4arc $(p<0.05)$. Compared with the Trilogy's plans, the Halcyon's plans reduced the high-dose volume of the heart and LV but increased the mean dose of the heart. For the dose of the LAD and the $V_{20}$ and $V_{30}$ of lungs, there was no significant difference between the two accelerators. Compared with the Trilogy, plans on the Halcyon significantly increased the skin dose but also significantly reduced the delivery time.

Conclusion: For the Halcyon, the whole-arc plans have more dosimetric advantages than partial-arc plans in bilateral breast cancer radiotherapy. Although the mean dose of the heart and the skin dose are increased, the doses of the cardiac substructure and other OARs are comparable to the Trilogy, and the delivery time is significantly reduced.

Keywords: Bilateral breast cancer, Volumetric modulated arc therapy, Halcyon, Trilogy, Dosimetry

*Correspondence: yinyongsd@126.com

${ }^{\dagger}$ Tao Sun and Xiutong Lin have contributed equally to this work Department of Radiation Physics and Technology, Shandong Cancer Hospital and Institute, Shandong First Medical University and Shandong Academy of Medical Sciences, 440 Jiyan Road, Jinan 250117, Shandong, China
Breast cancer is one of the most common malignant tumours in women. Postoperative radiotherapy can significantly reduce the recurrence rate of tumours, reduce mortality, and prolong the survival time of patients with breast cancer [1]. Bilateral breast cancer is relatively rare, accounting for approximately $2.1 \%$ of all patients with original author(s) and the source, provide a link to the Creative Commons licence, and indicate if changes were made. The images or other third party material in this article are included in the article's Creative Commons licence, unless indicated otherwise in a credit line to the material. If material is not included in the article's Creative Commons licence and your intended use is not permitted by statutory regulation or exceeds the permitted use, you will need to obtain permission directly from the copyright holder. To view a copy of this licence, visit http://creativecommons.org/licenses/by/4.0/. The Creative Commons Public Domain Dedication waiver (http://creativeco mmons.org/publicdomain/zero/1.0/) applies to the data made available in this article, unless otherwise stated in a credit line to the data. 
breast cancer [2]. Because of the larger target area and more complex shape, it is more difficult to design the treatment plan for bilateral breast cancer.

The Halcyon linear accelerator (LINAC) is a new machine from Varian company that offers a single $6 \mathrm{MV}$ flattening-filter-free (FFF) X-ray with a jawless design. The Halcyon has many differences from conventional C-arm LINACs, such as jawless design, a dual-layer multi-leaf collimator (MLC), faster gantry rotation and obligatory daily image-guided radiotherapy (IGRT). Comparable dose distribution and excellent delivery efficiency have been shown on the Halcyon in radiotherapy for head and neck, brain, unilateral breast, and cervical cancers [3-7]. Trilogy is a conventional C-arm LINAC with jaw and Millennium MLC.

It has been proven that volumetric modulated arc therapy (VMAT) can improve the quality of the plan and the delivery efficiency in bilateral breast cancer [810]. A previous study found that the partial-arc VMAT plans significantly reduced the cardiopulmonary dose compared with the whole-arc VMAT plans in unilateral breast radiotherapy [11]. Similarly, partial-arc VMAT plans were feasible in bilateral breast radiotherapy [12]. These studies were based on conventional C-arm LINAC with jaw. To our knowledge, applications of the Halcyon in the VMAT plans for bilateral breast cancer have not been reported. It is unknown whether the partial-arc VMAT plan is applicable on the Halcyon. With the Halcyon 1.0, high-quality/low-dose megavoltage cone beam computed tomography (MV CBCT) or orthogonal MV radiograph pair could be selected for image guidance, and the four setup-fields delivered different monitor units (MUs).

The aim of this study was to compare the dosimetric differences of the four setup-field plans and the suitable arc modes of VMAT plans for the Halcyon in bilateral breast radiotherapy and to analyse the plan quality and delivery efficiency by comparing the dosimetric differences between the Halcyon and the Trilogy to guide the clinical application in bilateral breast radiotherapy.

\section{Materials and methods}

\section{Patient selection and volume delineation}

From September 2006 to December 2018, CT image datasets of 10 patients diagnosed with bilateral breast cancer and who received bilateral breast radiotherapy at Shandong Cancer Hospital were selected. The clinical target volume (CTV) included all bilateral breast tissue, excluding local lymph node region. The planning target volume (PTV) was generated by expanding a 5 - $\mathrm{mm}$ margin from the CTV and was shrunk to $5 \mathrm{~mm}$ below the skin on the skin side. The organs at risk (OARs) include the total lung, heart, left ventricle (LV), left anterior descending artery (LAD) and liver. The skin is defined as the 3-mm region below the body outside of the PTV. The normal structures were defined as the body minus the PTV (B-P).

\section{Treatment planning}

With the Halcyon version 1.0, all imaging setup fields are taken using digital megavoltage imaging panels. When designing a Halcyon plan, four different setup fields can be selected. For the 10 patients, we designed four VMAT plans with different setup fields on the Halcyon: highquality MV CBCT (the gantry rotates clockwise from $260^{\circ}$ to $100^{\circ}$, delivering $10 \mathrm{MUs}$, simply called CBCT-H); low-dose MV CBCT (delivering 5 MUs in a clockwise gantry rotation from $260^{\circ}$ to $100^{\circ}$, simply called CBCTL); high-quality orthogonal MV radiograph pair (images acquired with $0^{\circ}$ and $90^{\circ}$ and delivering 2 MUs for each field, simply called $\mathrm{MV}-\mathrm{H}$ ) and low-dose orthogonal $\mathrm{MV}$ radiograph pair (images acquired with $0^{\circ}$ and $90^{\circ}$ and delivering $1 \mathrm{MU}$ for each field, simply called MV-L). For the four plans, two anticlockwise $160^{\circ}-200^{\circ}$ and two clockwise $200^{\circ}-160^{\circ}$ rotation arcs were used.

On the Trilogy, whole and partial arc plans, referred to as T-4arc and T-8arc, respectively, were generated. The whole-arc plan consisted of two anticlockwise $160^{\circ}-200^{\circ}$ and two clockwise $200^{\circ}-160^{\circ}$ rotation arcs. The partialarc plan consisted of total 8 partial arcs. For unilateral breast, four $100^{\circ}$ arcs like a bowknot were generated. In the two plans, the medial $\mathrm{x}$-jaw was set to the minimum site $(-2 \mathrm{~cm})$ to minimize the irradiated volume of the lungs and heart. A $6 \mathrm{MV}$ X-ray was used, and the dose rate was set to $600 \mathrm{MU} / \mathrm{min}$. Two whole and partial arc plans, referred to as $\mathrm{H}-4$ arc and $\mathrm{H}-8 \mathrm{arc}$, were designed on the Halcyon with the same arc angle as T-4arc and T-8arc mentioned above. In the Halcyon plans, a $6 \mathrm{MV}$ FFF X-ray was used at the maximum dose rate of 800 $\mathrm{MU} / \mathrm{min}$. Low-dose MV CBCT was selected for image guidance as Flores-Martinez et al. [6] suggested.

The prescription dose was 50 Gy in 2-Gy fractions. All plans were designed with the Eclipse version 15.5 treatment planning system (Varian Medical Systems, Palo Alto, CA, USA) using an analytic anisotropic algorithm (AAA). For all VMAT plans, the PTV was extended to $5 \mathrm{~mm}$ outside the skin, named PTVop, a $10-\mathrm{mm}$ bolus was used on the skin outside the PTV, and the dose of PTVop was optimized. In the final dose calculation, the bolus was deleted. The dose normalization was $95 \%$ volume of the PTV received 100\% prescription dose. All plans used the same optimization parameter settings, with the goal of minimizing the doses to the lungs, heart and LAD while ensuring PTV dose coverage. No dose constraints were applied to the skin and LV during the optimization. 


\section{Dosimetric evaluation}

The dose statistics of the plans were based on dose-volume histogram (DVH) analysis. For PTV, the dose of $2 \%$ and $98 \%$ volume $\left(D_{2}, D_{98}\right)$, the volume receiving $107 \%$ and $110 \%$ of the prescribed dose $\left(\mathrm{V}_{107}\right.$ and $\left.\mathrm{V}_{110}\right)$ and the mean dose $\left(\mathrm{D}_{\text {mean }}\right)$ were analysed. The conformity index (CI) and the homogeneity index (HI) of the PTV were calculated according to the following formula:

$$
C I=\frac{T V_{P V}^{2}}{T V \times P V}
$$

$\mathrm{TV}_{\mathrm{PV}}$ represents the volume of the PTV wrapped by the prescription dose, the TV represents the volume of the PTV, and the PV represents the total volume wrapped by the prescription dose. Larger CI values indicate the better conformity of the target [13].

$$
H I=\frac{D_{2}-D_{98}}{D_{p}} \times 100 \%
$$

$\mathrm{D}_{\mathrm{p}}$ represents the prescribed dose. Lower HI values indicate the better uniformity of the target [14].

For OARs, the $V_{X}$ and mean doses were analysed. $V_{X}$ represents the irradiated volume of $X$ Gy dose.

The number of MUs was analysed for all plans. The delivery times of T-4arc, T-8arc, $\mathrm{H}-4$ arc and $\mathrm{H}-8$ arc were recorded. The delivery time was recorded from the first field beam on to the last field beam off, excluding the positioning time.

\section{Statistical analysis}

All data were statistically analysed using the Statistical Package for Social Sciences v20.0 software (SPSS Inc., Chicago, IL, USA). First, a one-way analysis of variance (ANOVA) using Bonferroni's multiple comparisons test was applied to compare the four different setup-field plans on the Halcyon. Second, whole and partial arc plans on the same LINAC were compared to determine the most suitable field mode for the machine. The MannWhitney U test was used. The better Halcyon plan was selected for further comparisons. Third, a statistical comparison of the better Halcyon plan and the Trilogy plan was implemented to analyse the dosimetric differences between the two machines using the Mann-Whitney U test. The differences were considered statistically significant when $p<0.05$.

\section{Results}

\section{Dose comparisons for different setup-field plans}

Table 1 shows the dose parameters for the four different setup-field plans on the Halcyon. Among the four plans, no significant differences were observed in the
$\mathrm{D}_{98}, \mathrm{~V}_{110}, \mathrm{CI}$ and $\mathrm{HI}$ of the PTV. Compared to CBCT-L, CBCT-H plans increased the $\mathrm{D}_{\text {mean }}, \mathrm{D}_{2}$ and $\mathrm{V}_{107}$ of the PTV. For OARs, CBCT-H plans increased the $\mathrm{V}_{5}$ and $\mathrm{D}_{\text {mean }}$ of the heart, LV and lungs, $\mathrm{V}_{5}$ of LAD and B-P. For the skin, liver and MUs, there were no significant differences between the four plans. No significant differences were found among any of the dosimetric indicators of the PTV and OARs in the CBCT-L, MV-H and MV-L plans. CBCT-L plans showed the lowest values in the mean dose of heart, LAD, LV, lungs, and liver compared to MV-H and MV-L plans, but it was not statistically significant.

\section{Dose comparisons for different arc plans on identical LINACs}

Table 2 shows the PTV dosimetric parameters of the four whole and partial arc plans on the Halcyon and Trilogy LINACs. For PTV, there were no statistically significant differences in $\mathrm{D}_{\text {mean }}, \mathrm{D}_{2}, \mathrm{D}_{98}, \mathrm{~V}_{107}, \mathrm{~V}_{110}$ and $\mathrm{HI}$ between whole and partial arc plans on the Trilogy $(p<0.05)$. T-4arc showed better conformity than T-8arc $(p<0.05)$. On the Halcyon, the $\mathrm{D}_{2}, \mathrm{~V}_{107}, \mathrm{~V}_{110}$ and $\mathrm{HI}$ of the PTV in $\mathrm{H}-8$ arc were higher than those in $\mathrm{H}$-4arc $(p<0.05)$. The $\mathrm{D}_{98}$ and $\mathrm{CI}$ in $\mathrm{H}$-4arc plans were higher than those in H-8arc plans, and the differences were statistically significant. There was no significant difference in the $D_{\text {mean }}$ of the PTV between the two plans on the Halcyon.

Table 3 shows the dosimetric parameters of OARs. On the Trilogy, T-8arc plans significantly reduced the $V_{5}$ and $D_{\text {mean }}$ of the heart, the $V_{5}$ of $L V, V_{5}, V_{10}$ and $\mathrm{D}_{\text {mean }}$ of the lungs, the $\mathrm{D}_{\text {mean }}$ of the liver and total MUs compared with T-4arc. T-4arc plans reduced the $\mathrm{V}_{40}$ of the LV and lungs compared with T-8arc. No significant differences were observed in the doses of the LAD and skin and the delivery time. On the Halcyon, lung doses $\left(\mathrm{V}_{5}\right.$ and $\left.\mathrm{V}_{40}\right)$, heart doses $\left(\mathrm{V}_{5}, \mathrm{~V}_{20}, \mathrm{~V}_{30}, \mathrm{~V}_{40}\right.$ and $\left.\mathrm{D}_{\text {mean }}\right)$, and $\operatorname{LV}\left(\mathrm{V}_{20}, \mathrm{~V}_{30}, \mathrm{~V}_{40}\right.$ and $\left.\mathrm{D}_{\text {mean }}\right)$ and LAD $\left(\mathrm{V}_{30}, \mathrm{~V}_{40}\right.$, $\mathrm{D}_{\text {max }}$, and $\mathrm{D}_{\text {mean }}$ ) doses were significantly reduced in 4arc plans compared with 8 arc plans $(p<0.05)$. H-8arc plans significantly reduced the number of MUs compared with $\mathrm{H}$-4arc plans. No significant differences were observed in the skin dose and the delivery time. Figure 1 shows the mean dose-volume histograms for the four plans.

According to the results above, of all studied plans $\mathrm{T}-8$ arc and H-4arc plans showed the best dosimetry on the respective LINAC. Because of the poor dosimetry, H-8arc was not used for further statistical comparison. Next, H-4arc was statistically analysed with T-4arc and T-8arc, respectively, to reflect the dosimetric differences between the two LINACs. 
Table 1 Dosimetric analysis for four different setup-field plans on the Halcyon

\begin{tabular}{|c|c|c|c|c|c|c|}
\hline & CBCT-H & CBCT-L & MV-H & MV-L & $p$ & $p<0.05$ \\
\hline \multicolumn{7}{|l|}{ PTV } \\
\hline$D_{\text {mean }}(G y)$ & $52.4 \pm 0.3$ & $52.0 \pm 0.2$ & $52.2 \pm 0.2$ & $52.1 \pm 0.3$ & 0.01 & \multirow[t]{2}{*}{ a } \\
\hline $\mathrm{D}_{98}(\mathrm{~Gy})$ & $48.5 \pm 0.2$ & $48.5 \pm 0.3$ & $48.5 \pm 0.3$ & $48.6 \pm 0.4$ & 0.95 & \\
\hline$D_{2}(G y)$ & $54.3 \pm 0.60$ & $53.6 \pm 0.3$ & $53.8 \pm 0.4$ & $53.7 \pm 0.5$ & 0.01 & $a, c$ \\
\hline$V_{107}(\%)$ & $16.1 \pm 12.8$ & $3.6 \pm 2.8$ & $7.9 \pm 6.7$ & $7.5 \pm 10.8$ & 0.03 & a \\
\hline$V_{110}(\%)$ & $0.8 \pm 1.6$ & $0.0 \pm 0.0$ & $0.1 \pm 0.1$ & $0.0 \pm 0.1$ & 0.09 & \\
\hline $\mathrm{Cl}$ & $0.86 \pm 0.02$ & $0.86 \pm 0.02$ & $0.86 \pm 0.02$ & $0.86 \pm 0.02$ & 0.96 & \\
\hline $\mathrm{HI}$ & $11.6 \pm 1.3$ & $10.1 \pm 1.1$ & $10.6 \pm 1.1$ & $10.1 \pm 1.4$ & 0.06 & \\
\hline \multicolumn{7}{|l|}{ Heart } \\
\hline$V_{5}(\%)$ & $55.0 \pm 9.3$ & $30.5 \pm 6.2$ & $33.2 \pm 8.4$ & $29.4 \pm 7.7$ & 0.00 & \multirow[t]{2}{*}{$a, b, c$} \\
\hline $\mathrm{V}_{30}(\%)$ & $1.1 \pm 0.6$ & $0.8 \pm 0.5$ & $1.0 \pm 0.7$ & $1.1 \pm 0.7$ & 0.62 & \\
\hline $\mathrm{D}_{\text {mean }}(\mathrm{Gy})$ & $7.1 \pm 0.7$ & $5.6 \pm 0.6$ & $6.0 \pm 0.9$ & $5.7 \pm 0.9$ & 0.00 & $a, b, c$ \\
\hline \multicolumn{7}{|l|}{ LV } \\
\hline $\mathrm{V}_{5}(\%)$ & $57.9 \pm 11.2$ & $35.7 \pm 9.3$ & $39.9 \pm 11.6$ & $36.0 \pm 10.4$ & 0.00 & \multirow[t]{2}{*}{$a, b, c$} \\
\hline$V_{30}(\%)$ & $2.6 \pm 1.3$ & $1.9 \pm 1.0$ & $2.5 \pm 1.5$ & $2.6 \pm 1.5$ & 0.56 & \\
\hline$D_{\text {mean }}(G y)$ & $8.7 \pm 1.3$ & $6.8 \pm 1.1$ & $7.3 \pm 1.5$ & $6.9 \pm 1.4$ & 0.03 & $a, c$ \\
\hline \multicolumn{7}{|l|}{$L A D$} \\
\hline$V_{5}(\%)$ & $97.5 \pm 5.1$ & $84.6 \pm 13.4$ & $85.1 \pm 12.7$ & $80.6 \pm 14.5$ & 0.02 & \multirow[t]{4}{*}{ c } \\
\hline$V_{30}(\%)$ & $21.3 \pm 13.8$ & $14.3 \pm 10.9$ & $20.8 \pm 15.0$ & $21.6 \pm 15.0$ & 0.59 & \\
\hline$D_{\max }(G y)$ & $39.5 \pm 3.2$ & $38.9 \pm 3.1$ & $39.7 \pm 3.3$ & $39.8 \pm 3.5$ & 0.92 & \\
\hline $\mathrm{D}_{\text {mean }}(\mathrm{Gy})$ & $19.2 \pm 4.7$ & $17.2 \pm 4.0$ & $18.5 \pm 4.6$ & $18.2 \pm 5.0$ & 0.80 & \\
\hline \multicolumn{7}{|l|}{ Lungs } \\
\hline$V_{5}(\%)$ & $57.7 \pm 8.9$ & $43.7 \pm 4.1$ & $46.6 \pm 7.4$ & $43.7 \pm 6.6$ & 0.00 & \multirow[t]{2}{*}{$a, b, c$} \\
\hline $\mathrm{V}_{20}(\%)$ & $14.9 \pm 2.9$ & $13.2 \pm 2.5$ & $14.7 \pm 2.7$ & $14.2 \pm 2.4$ & 0.47 & \\
\hline $\mathrm{D}_{\text {mean }}(\mathrm{Gy})$ & $10.4 \pm 1.1$ & $9.1 \pm 0.8$ & $9.6 \pm 1.0$ & $9.3 \pm 0.9$ & 0.03 & a \\
\hline \multicolumn{7}{|l|}{ Liver } \\
\hline$D_{\text {mean }}(G y)$ & $6.9 \pm 1.5$ & $6.0 \pm 1.1$ & $6.5 \pm 1.5$ & $6.3 \pm 1.4$ & 0.54 & \\
\hline \multicolumn{7}{|l|}{ Skin } \\
\hline$V_{40}(\%)$ & $41.3 \pm 9.2$ & $40.4 \pm 8.8$ & $39.7 \pm 9.2$ & $39.2 \pm 8.5$ & 0.96 & \\
\hline$V_{50}(\%)$ & $0.3 \pm 0.2$ & $0.3 \pm 0.2$ & $0.1 \pm 0.1$ & $0.1 \pm 0.1$ & 0.12 & \\
\hline \multicolumn{7}{|l|}{ B-P } \\
\hline$V_{5}(\%)$ & $32.9 \pm 4.5$ & $27.6 \pm 2.8$ & $28.9 \pm 3.5$ & $28.0 \pm 3.4$ & 0.01 & \multirow[t]{3}{*}{$a, c$} \\
\hline$V_{10}(\%)$ & $19.9 \pm 2.3$ & $18.4 \pm 1.9$ & $19.4 \pm 2.0$ & $19.0 \pm 2.1$ & 0.44 & \\
\hline MUs & $936.5 \pm 68.2$ & $1036.1 \pm 103.8$ & $956.6 \pm 73.4$ & $986.2 \pm 76.5$ & 0.05 & \\
\hline
\end{tabular}

a: CBCT-H versus CBCT-L; b: CBCT-H versus MV-H; c: CBCT-H versus MV-L; d: CBCT-L versus MV-H; e: CBCT-L versus MV-L; f: MV-H versus MV-L

Table 2 Dosimetric parameters of PTV for whole and partial arc plans on the Trilogy and Halcyon

\begin{tabular}{lccccl}
\hline PTV & T-4arc & T-8arc & H-4arc & H-8arc & $P<\mathbf{0 . 0 5}$ \\
\hline $\mathrm{D}_{\text {mean }}(\mathrm{Gy})$ & $51.8 \pm 0.2$ & $51.6 \pm 0.3$ & $52.0 \pm 0.2$ & $52.2 \pm 0.2$ & $\mathrm{c}, \mathrm{d}$ \\
$\mathrm{D}_{2}(\mathrm{~Gy})$ & $53.5 \pm 0.4$ & $53.1 \pm 0.6$ & $53.6 \pm 0.31$ & $54.2 \pm 0.47$ & $\mathrm{~b}, \mathrm{c}$ \\
$\mathrm{D}_{98}(\mathrm{~Gy})$ & $48.4 \pm 0.4$ & $48.0 \pm 0.6$ & $48.5 \pm 0.3$ & $48.1 \pm 0.4$ & $\mathrm{~b}, \mathrm{c}$ \\
$\mathrm{V}_{107}(\%)$ & $3.2 \pm 2.4$ & $1.4 \pm 2.2$ & $3.6 \pm 2.8$ & $11.1 \pm 8.4$ & $\mathrm{~b}, \mathrm{c}$ \\
$\mathrm{V}_{110}(\%)$ & $0.0 \pm 0.1$ & $0.0 \pm 0.0$ & $0.0 \pm 0.0$ & $0.4 \pm 0.5$ & $\mathrm{~b}$ \\
$\mathrm{Cl}$ & $0.87 \pm 0.02$ & $0.82 \pm 0.04$ & $0.86 \pm 0.02$ & $0.83 \pm 0.03$ & $\mathrm{a}, \mathrm{b}, \mathrm{c}$ \\
$\mathrm{HI}(\%)$ & $10.3 \pm 1.5$ & $10.2 \pm 2.1$ & $10.1 \pm 1.1$ & $12.0 \pm 1.4$ & $\mathrm{~b}$ \\
\hline
\end{tabular}

a: T-4arc versus T-8arc, b: $\mathrm{H}-4$ arc versus $\mathrm{H}-8$ arc, c: T-8arc versus $\mathrm{H}-4$ arc, d: T-4arc versus $\mathrm{H}-4$ arc
Dose comparisons for the plans between the trilogy and the halcyon

The imaging dose of Trilogy is not calculated into the entire plan in contrast to Halcyon plans. In Tables 2 and 3, the doses of PTV and OARs on Trilogy were only caused by the treatment field, without considering the additional effect of the imaging field. For the PTV, a significant difference was observed in the $D_{\text {mean }}$ between $\mathrm{T}$-4arc and $\mathrm{H}$-4arc. There were no statistically significant differences in the $\mathrm{D}_{2}, \mathrm{D}_{98}, \mathrm{~V}_{107}, \mathrm{~V}_{110}$, and $\mathrm{CI}$, HI between the two plans. Compared with T-8arc plan, H-4arc significantly increased the $\mathrm{D}_{\text {mean }}, \mathrm{D}_{2}$ and $\mathrm{V}_{107}$ of the PTV. 
Table 3 Dosimetric parameters of OARs and delivery efficiency for whole and partial arc plans on the Trilogy and Halcyon

\begin{tabular}{|c|c|c|c|c|c|}
\hline & T-4arc & T-8arc & H-4arc & H-8arc & $p<0.05$ \\
\hline \multicolumn{6}{|l|}{ Heart } \\
\hline$V_{5}(\%)$ & $20.4 \pm 6.2$ & $13.7 \pm 4.9$ & $30.5 \pm 6.2$ & $49.2 \pm 4.5$ & $a, b, c, d$ \\
\hline$V_{10}(\%)$ & $8.5 \pm 3.3$ & $8.0 \pm 3.7$ & $10.2 \pm 2.9$ & $15.0 \pm 6.4$ & - \\
\hline$V_{20}(\%)$ & $3.4 \pm 1.5$ & $4.0 \pm 2.4$ & $2.7 \pm 1.3$ & $5.8 \pm 4.0$ & $b$ \\
\hline$V_{30}(\%)$ & $1.1 \pm 0.6$ & $1.8 \pm 1.7$ & $0.8 \pm 0.5$ & $2.7 \pm 2.8$ & $b, c$ \\
\hline$V_{40}(\%)$ & $0.2 \pm 0.2$ & $0.6 \pm 0.9$ & $0.1 \pm 0.1$ & $1.1 \pm 1.8$ & $b, c$ \\
\hline$D_{\text {mean }}(G y)$ & $4.9 \pm 0.6$ & $3.9 \pm 1.1$ & $5.6 \pm 0.6$ & $7.1 \pm 1.6$ & $a, b, c, d$ \\
\hline \multicolumn{6}{|l|}{ LV } \\
\hline$V_{5}(\%)$ & $34.2 \pm 10.3$ & $23.9 \pm 7.6$ & $35.7 \pm 9.3$ & $48.9 \pm 14.8$ & $a, c$ \\
\hline$V_{10}(\%)$ & $17.5 \pm 6.6$ & $15.5 \pm 6.6$ & $16.1 \pm 5.9$ & $22.9 \pm 9.8$ & - \\
\hline$V_{20}(\%)$ & $7.6 \pm 3.0$ & $9.1 \pm 5.2$ & $5.8 \pm 2.7$ & $12.0 \pm 7.7$ & $b$ \\
\hline$V_{30}(\%)$ & $2.7 \pm 1.3$ & $4.7 \pm 4.0$ & $1.9 \pm 1.0$ & $6.5 \pm 6.2$ & $b, c$ \\
\hline$V_{40}(\%)$ & $0.5 \pm 0.5$ & $1.6 \pm 2.0$ & $0.2 \pm 0.1$ & $2.9 \pm 4.1$ & $a, b, c$ \\
\hline$D_{\text {mean }}(G y)$ & $6.9 \pm 1.3$ & $6.0 \pm 2.0$ & $6.8 \pm 1.1$ & $9.2 \pm 3.0$ & b \\
\hline \multicolumn{6}{|l|}{ LAD } \\
\hline$V_{5}(\%)$ & $85.7 \pm 14.5$ & $76.0 \pm 11.4$ & $84.6 \pm 13.4$ & $89.3 \pm 8.7$ & - \\
\hline$V_{10}(\%)$ & $61.6 \pm 16.5$ & $63.6 \pm 16.9$ & $63.2 \pm 17.2$ & $67.5 \pm 16.6$ & - \\
\hline$V_{20}(\%)$ & $43.3 \pm 17.7$ & $47.5 \pm 21.8$ & $43.1 \pm 19.1$ & $52.9 \pm 21.7$ & - \\
\hline$V_{30}(\%)$ & $24.2 \pm 15.5$ & $25.7 \pm 20.5$ & $14.3 \pm 10.9$ & $31.2 \pm 18.2$ & $b$ \\
\hline$V_{40}(\%)$ & $1.2 \pm 1.5$ & $6.5 \pm 15.4$ & $0.1 \pm 0.3$ & $9.8 \pm 15.9$ & $b$ \\
\hline$D_{\max }(G y)$ & $40.4 \pm 4.2$ & $41.9 \pm 4.2$ & $38.9 \pm 3.1$ & $42.5 \pm 4.4$ & $b$ \\
\hline $\mathrm{D}_{\text {mean }}(\mathrm{Gy})$ & $18.1 \pm 4.7$ & $19.0 \pm 6.3$ & $17.2 \pm 4.0$ & $21.3 \pm 5.8$ & $b$ \\
\hline \multicolumn{6}{|l|}{ Lungs } \\
\hline$V_{5}(\%)$ & $45.1 \pm 3.1$ & $30.4 \pm 3.5$ & $43.7 \pm 4.1$ & $49.1 \pm 4.7$ & $a, b, c$ \\
\hline$V_{10}(\%)$ & $23.9 \pm 2.6$ & $19.4 \pm 2.6$ & $22.9 \pm 2.6$ & $25.5 \pm 2.9$ & $a, c$ \\
\hline$V_{20}(\%)$ & $13.4 \pm 2.2$ & $12.4 \pm 2.6$ & $13.2 \pm 2.5$ & $15.8 \pm 3.0$ & - \\
\hline$V_{30}(\%)$ & $7.9 \pm 1.8$ & $8.5 \pm 2.3$ & $8.0 \pm 2.1$ & $10.6 \pm 3.0$ & - \\
\hline$V_{40}(\%)$ & $3.2 \pm 1.1$ & $5.0 \pm 1.9$ & $3.5 \pm 1.4$ & $6.0 \pm 2.5$ & $a, b$ \\
\hline$D_{\text {mean }}(G y)$ & $9.1 \pm 0.7$ & $7.5 \pm 1.0$ & $9.1 \pm 0.8$ & $9.2 \pm 3.0$ & $a, c$ \\
\hline \multicolumn{6}{|l|}{ Skin } \\
\hline$V_{30}(\%)$ & $67.3 \pm 8.7$ & $68.9 \pm 8.2$ & $74.2 \pm 7.6$ & $75.2 \pm 6.9$ & $c, d$ \\
\hline$V_{40}(\%)$ & $31.3 \pm 7.9$ & $32.4 \pm 8.5$ & $40.4 \pm 8.8$ & $41.5 \pm 7.3$ & $c, d$ \\
\hline$V_{45}(\%)$ & $7.9 \pm 3.7$ & $9.3 \pm 3.7$ & $16.8 \pm 4.3$ & $17.5 \pm 3.3$ & $c, d$ \\
\hline$V_{50}(\%)$ & $0.0 \pm 0.1$ & $0.0 \pm 0.0$ & $0.3 \pm 0.2$ & $0.5 \pm 0.5$ & $c, d$ \\
\hline$D_{\text {mean }}(G y)$ & $33.9 \pm 2.0$ & $34.3 \pm 1.9$ & $36.0 \pm 1.9$ & $36.3 \pm 1.6$ & $c, d$ \\
\hline \multicolumn{6}{|l|}{ Liver } \\
\hline$D_{\text {mean }}(G y)$ & $5.7 \pm 1.5$ & $3.9 \pm 1.1$ & $6.0 \pm 1.1$ & $6.7 \pm 1.5$ & $a, c$ \\
\hline $\mathrm{MU}$ & $1170.8 \pm 97.3$ & $1051.5 \pm 70.1$ & $1036.1 \pm 103.8$ & $831.6 \pm 45.2$ & $a, b, d$ \\
\hline Delivery time (min) & $6.1 \pm 0.6$ & $6.0 \pm 0.9$ & $2.2 \pm 0.2$ & $2.2 \pm 0.4$ & $c, d$ \\
\hline
\end{tabular}

a: T-4arc versus T-8arc, b: $\mathrm{H}$-4arc versus $\mathrm{H}-8$ arc, c: T-8arc versus $\mathrm{H}-4 a r c, \mathrm{~d}$ : T-4arc versus $\mathrm{H}$-4arc

Better $\mathrm{D}_{98}$ and CI were observed in H-4arc. No statistically significant differences were observed in the $\mathrm{V}_{110}$ and $\mathrm{HI}$ between the T-8arc and $\mathrm{H}-4$ arc plans. The results are shown in Table 2.

For the doses of heart and substructures, $\mathrm{H}$-4arc plans increased the $V_{5}$ and $D_{\text {mean }}$ of the heart but reduced the $\mathrm{V}_{30}$ and $\mathrm{V}_{40}$ of the heart and LV $(p<0.05)$. No statistically significant differences were found in the $V_{10}$ and
$\mathrm{V}_{20}$ of the heart, the $\mathrm{V}_{10}, \mathrm{~V}_{20}$ and $\mathrm{D}_{\text {mean }}$ of the LV, and all dosimetric parameters of the LAD between $\mathrm{H}$-4arc and the two Trilogy plans. H-4arc plans showed the lowest values in the heart and $L V$ doses $\left(\mathrm{V}_{20}, \mathrm{~V}_{30}, \mathrm{~V}_{40}\right)$ and the LAD doses $\left(\mathrm{V}_{30}, \mathrm{~V}_{40}, \mathrm{D}_{\text {max }}, \mathrm{D}_{\text {mean }}\right)$ compared with the two Trilogy plans, but some data showed no statistical significance (Table 3). T-8arc plans significantly reduced the $V_{5}, V_{10}$ and $D_{\text {mean }}$ of the lungs and the $D_{\text {mean }}$ of the 

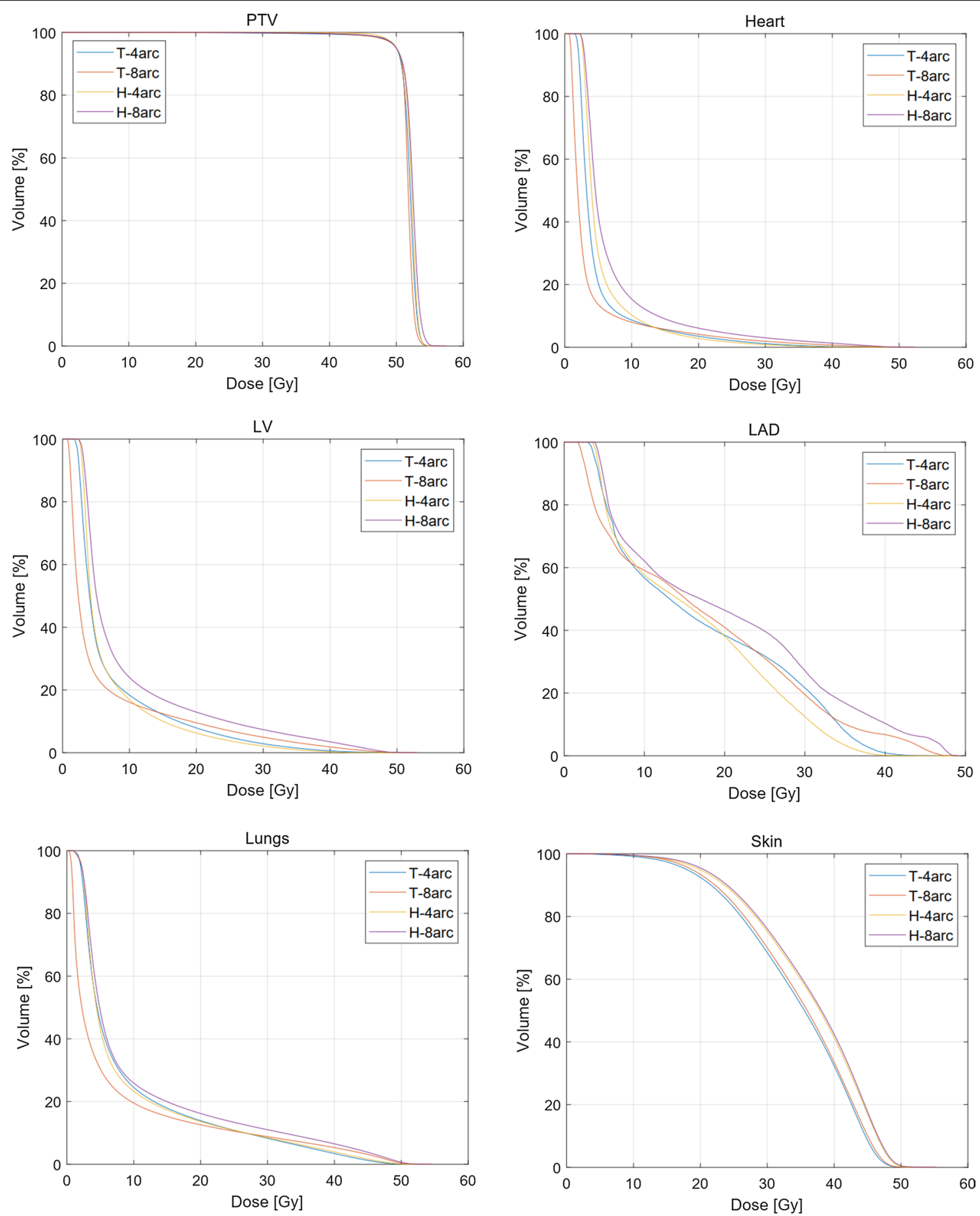

Fig. 1 Mean dose-volume histograms for the four plans

liver compared with T-4arc and H-4arc plans, and no statistically significant differences were found in these indexes between the latter two plans. For the $V_{20}, V_{30}$, and $V_{40}$ of the lungs, there were no statistically significant differences between $\mathrm{H}-4 \mathrm{arc}$ and the two Trilogy plans $(p>0.05) . \mathrm{H}-4$ arc plans increased the skin doses $\left(\mathrm{V}_{30}\right.$, $\mathrm{V}_{40}, \mathrm{~V}_{45}, \mathrm{~V}_{50}$, and $\mathrm{D}_{\text {mean }}$ ) compared with the two Trilogy plans.

T-4arc plans showed the largest number of MUs compared with the T-8arc and H-4arc plans, and no 
statistically significant difference was found between the latter two plans. The average delivery times of T-4arc, $\mathrm{T}$-8arc, and $\mathrm{H}$-4arc were $6.05 \pm 0.57,6.04 \pm 0.89$, and $2.18 \pm 0.15$ min, respectively. $\mathrm{H}-4$ arc plans showed the shortest delivery times (Table 3).

\section{Discussion}

In radiotherapy for breast cancer, high position accuracy is essential to prevent under-dose in the target and excessive irradiation to OARs. Image guidance must be taken before each patient is treated on the Halcyon. Compared to two-dimensional (2D) position verification, threedimensional (3D) position verification like CBCT can more accurately measure $3 \mathrm{D}$ vector changes and observe the body position rotation $[15,16]$. For breast tissue, CBCT is very useful because it can provide $3 \mathrm{D}$ soft tissue and bony anatomy information and can be compared with the planning $\mathrm{CT}$ to assess the accuracy of the setting [16, 17]. Rossi et al. [18] reported that CBCT matching is recommended when breast/chest wall patients were treated with the VMAT technique. However, CBCT may be associated with more additional dose and time-consuming than orthogonal planar images. On Halcyon, MV CBCT imaging process take only $15 \mathrm{~s}$. It's beneficial for patients. In C-arm accelerators such as the Trilogy, kilovoltage (KV) CBCT can be used to correct the patient's position if $3 \mathrm{D}$ position verification is necessary. In Halcyon 1.0, MV CBCT was used for image-guided radiotherapy (IGRT). Compared to KV imaging, MV imaging has some advantages, such as identical isocenter as the treatment beam and no metal artifacts. Compared with $\mathrm{KV}$ imaging, the main disadvantages of MV imaging are higher dose and lower image quality. About Halcyon's MV CBCT, Malajovich et al. [19] reported that the highest tissue dose of MV CBCT ranges from 2 to 7 cGy per fraction in different treatment sites, which is equivalent to the fractional dose of KV CBCT during breast and pelvic IGRT application, and MV CBCT images of Halcyon is able to identify different soft tissues and lack of metal-induced artifacts. On Halcyon, two different dose image mode could be selected to apply the CBCT. Compared to the low-dose mode, the high-quality imaging mode does not provide material advantages [19]. We found that high-quality CBCT plans increased the OARs doses compared to low-dose CBCT plans. Therefore, we inferred that low-dose MV CBCT was the optimal setupfield mode. Flores-Martinez et al. [6] compared four different setup-field plans for unilateral breast cancer, and in their opinion, low-dose MV CBCT was the most suitable technique for patients treated on the Halcyon. Their results agreed with ours. On Halcyon, the dose of the setup field is incorporated in the calculation of the planned dose, and the irradiated doses to the target and the OARs are also reflected in the total plan dose, which is more intuitive.

Partial-arc plans on the Trilogy showed more dosimetric advantage, especially in low-dose volumes of the heart, left ventricle, and lungs and the mean doses of the heart, lungs, and liver. This is because while designing a partial-arc plan, it is possible to artificially choose the arc degree that irradiates less OAR volume. Rotating the collimator angle and fixing the jaw can further reduce the influence of the leakage between the MLC on the dose of the OARs, which can minimize the dose of the OARs. Boman et al. [11] compared the dosimetric differences between the whole and partial arc VMAT plans of unilateral breast cancer, including regional lymph node irradiation, and the results showed that partial-arc plans significantly reduced the dose of the ipsilateral lung and the $V_{5}$ of the heart but increased the $V_{5}$ of the contralateral breast. The result is similar to ours, but the cases in our study are bilateral breast cancer, which does not involve the dose of the contralateral breast. Comparing the two plans of the Halcyon, the results were contrary to the Trilogy's, and the whole-arc plans showed better dosimetry. For the PTV, in addition to the mean dose, the whole-arc plan was better than partial-arc plans in terms of the maximum dose, minimum dose, conformity and uniformity. For OARs, partial-arc plans increased the doses to the heart, LV, LAD, and lungs. The results showed that partial-arc plans have no advantage for the Halcyon, which may be related to the jawless setting and the fewer arc degrees. According to the results described above, when designing a treatment plan for bilateral breast cancer, we can choose a more suitable arc setting according to the corresponding LINAC.

Based on the results described above, we mainly compared the dosimetric differences between the Halcyon's whole-arc plan and the Trilogy's two plans. All plans met clinical requirements. For the PTV, apart from the mean dose, there were no significant differences in other dosimetric parameters between the two whole-arc plans on the Halcyon and the Trilogy. Compared with the T-8arc plan, $\mathrm{H}$-4arc showed worse $\mathrm{D}_{\text {mean }}, \mathrm{D}_{2}$ and $\mathrm{V}_{107}$, and better $\mathrm{D}_{98}$ and CI of the PTV. In short, the plans of the two machines were comparable in terms of target dose.

Darby et al. [20] found a linear relationship between the mean dose of the heart and the incidence of ischaemic heart disease, and the incidence increased by $7.4 \%$ for each $1-$ Gy increase in the mean dose. Therefore, the mean dose of the heart is often used as a reference for cardiac toxicity. However, the dose to the cardiac substructure also needs to be considered in radiotherapy. Some studies believe that the LAD and LV are important parts of the heart in the context of radiation-induced heart disease [21-23]. In this study, 
for low-dose irradiated volumes of the heart and LV, partial-arc plans on the Trilogy showed the lowest values, while for high-dose irradiated volumes, the wholearc plans on the Halcyon showed the lowest values. For the mean dose of the heart, the partial-arc plans on the Trilogy showed the lowest value, and the whole-arc plans on the Halcyon showed the highest values. This may be related to the additional radiation dose to the heart from each MV CBCT scan. For all dosimetric parameters of the LAD, there is no significant difference between whole-arc plans on the Halcyon and the two plans on the Trilogy.

For lungs, the partial-arc plans on the Trilogy reduced the low-dose irradiated volume $\left(\mathrm{V}_{5}, \mathrm{~V}_{10}\right)$ and the mean dose but increased the high-dose irradiated volume $\left(\mathrm{V}_{40}\right)$. There was no significant difference in the comparison of the $V_{20}$ and $V_{30}$ in the lungs between all plans of the two LINACs. For all dosimetric parameters of the lungs, there was no significant difference between the two whole-arc plans on the two LINACs. Fiorentino et al. [24] retrospectively analysed the VMAT plans of 16 patients with bilateral breast cancer. For lungs, the average values of the $D_{\text {mean }}, V_{5}$ and $V_{20}$ were $11.8 \pm 2.3 \mathrm{~Gy}, 78.9 \pm 15.3 \%$ and $15.7 \pm 5 \%$, respectively. No acute and late complications above grade 2 were observed during the 24 months of follow-up. In our study, the mean dose, $V_{5}$ and $V_{20}$ of the lungs in all plans were lower than in their study.

The plans on the Halcyon increased the skin's dose compared to the two plans on the Trilogy. O'Grady et al. [25] found that 6 X FFF fields on the Halcyon increased the superficial dose compared to FF fields for breast cancer radiotherapy, as demonstrated by in vivo measurements, phantom measurements, and planning comparisons. Their results were the same as ours. Because the rays are softened after the flattening filter is removed, the $6 \mathrm{MV}$ X-rays in FFF mode are equivalent to lowerenergy rays, resulting in a shallower depth of the dose build-up area, thereby increasing the superficial dose. Studies on the Monte Carlo (MC) have shown that the influence of contamination electrons in the FFF mode is greater, which further leads to an increase in surface dose [26, 27]. Barsky et al. [5] retrospectively analysed 34 breast cancer cases treated on the Halcyon, and the results showed that breast cancer cases were well tolerated on Halcyon; additionally, the acute toxicity was comparable to the published reports using conventional LINACs. The difference from our study is that they used tangential fields instead of the VMAT in our study. On the Halcyon, the effect of VMAT on the skin dose needs more prospective clinical studies to be proved.

In contrast to Halcyon, KV CBCT could be used for image-guided on Trilogy. Although the dose of $\mathrm{KV}$ CBCT is less than that of MV CBCT, it is not negligible.
The difference with Halcyon is that the imaging dose of Trilogy is not calculated into the entire plan. So, in fact, the dose difference between the two machines may vary slightly. The whole-arc plans on Halcyon reduced the number of MUs compared to whole-arc plans on Trilogy and showed similar MUs compared to partial-arc plans on Trilogy. On Halcyon, the imaging dose is integrated into plans and thus it is contributing to the PTV and OAR dose. However, in Trilogy the imaging dose is not accounted for plans, what might explain the lower MUs required on Halcyon.

Previous studies have demonstrated that the Halcyon could reduce the treatment time significantly compared with conventional LINACs [3-7]. Our study proved the same results in radiotherapy for bilateral breast cancer. This may be related to the Halcyon's faster gantry and MLC speed and higher dose rate. The shortening of the treatment time can reduce intra-fraction movement, improve patient comfort, and increase machine throughput.

An issue that needs to be addressed is the correction of rotation errors. On C-arm accelerators like Trilogy, we can use the six degree of freedom (6-DoF) couch to correct the rotation errors. The combination of 6-DoF couch and $\mathrm{CBCT}$ can correct the translational and rotational setup errors and improve the positioning accuracy obviously [28, 29]. However, on O-ring accelerators like Halcyon, the installed 3-DoF couch allows only for correction of translational shifts. On the Halcyon, if large rotation errors are found, time-consuming repositioning may be required to improve the positioning accuracy. This drawback might significantly compromise the advantage of faster treatment plan delivery with Halcyon compared to Trilogy. Therefore, in order to reduce the positioning time and improve the positioning accuracy, it is necessary to improve the immobilization equipment or adopt online 3D IGRT equipment, such as surface imaging. 3D surface imaging system is a quick and non-invasive method to assist the patient in setting up, which could improve the accuracy and speed of patient positioning in breast cancer radiotherapy [30, 31]. On Halcyon, 3D surface imaging is especially suitable, which could correct rotation errors in patient positioning and monitor patient movement during beam delivery [32]. The combination of 3D surface imaging and daily CBCT may greatly improve treatment accuracy and reduce positioning time, which needs more research to prove in the future.

Many studies [33-35] have shown that deep inspiration breath hold (DIBH) can significantly reduce the radiation dose to the heart and lungs for breast cancer radiotherapy. However, the technique requires patients to hold their breath for a certain amount of time. Minimizing the 
treatment time is beneficial to patients with DIBH. The combination of Halcyon and VMAT can further reduce the treatment time and expand the application range of DIBH. The robustness of VMAT plans under respiratory motion or other influences which might change form or position of PTV needs to be considered. Field-in-field and fixed-field intensity-modulated radiation therapy can reduce the influence of respiratory movement and skin deformation or swelling during treatment by opening the MLC outside the skin or adding skin flash. When designing VMAT plans, we could increase the robustness of the plan by adding a virtual bolus on the skin $[11,36,37]$.

\section{Conclusions}

VMAT plans on the Halcyon can meet the clinical requirements in radiotherapy for bilateral breast cancer. Low-dose MV CBCT can be selected as an optimal setup mode. For Halcyon, the whole-arc plans are better than the partial-arc plans. While the Halcyon increases the mean dose to the heart and the dose to the skin compared with the conventional LINAC, it is comparable to the Trilogy in doses to the cardiac substructure and other OARs and significantly reduces the delivery time.

\begin{abstract}
Abbreviations
LINAC: Linear accelerator; FFF: Flattening-filter-free; MLC: Multi-leaf collimator; IGRT: Image guided radiotherapy; VMAT: Volumetric modulated arc therapy; MV CBCT: Megavoltage cone beam computed tomography; MUs: Monitor units; CTV: Clinical target volume; PTV: Planning target volume; OARs: Organs at risk; LV: Left ventricle; LAD: Left anterior descending artery; B-P: Body minus the PTV; DMI: Digital megavoltage imaging; AAA: Analytic anisotropic algorithm; Cl: Conformity index; HI: Homogeneity index; ANOVA: One-way analysis of variance; CBCT-H: Plan with high-quality MV CBCT; CBCT-L: Plan with low-dose MV CBCT; MV-H: Plan with high-quality MV pair; MV-L: Plan with low-dose MV pair; T-4arc: Whole-arc plans on Trilogy; T-8arc: Partial-arc plans on Trilogy; H-4arc: Whole-arc plans on Halcyon; H-8arc: Partial-arc plans on Halcyon; MC: Monte Carlo; DIBH: Deep inspiration breath hold.
\end{abstract}

\section{Acknowledgements}

Not applicable.

\section{Authors' contributions}

TS and YY designed the study. GZ collected the CT data. XL and TS designed the treatment plans. QQ and $C L$ analyzed the data. TS and XL wrote the paper. All authors read and approved the final manuscript.

\section{Funding}

This study was supported by the Natural Science Foundation of Shandong Province (Grant Nos. ZR2019LZL017 and ZR2020QH198), the Taishan Scholars Project of Shandong Province (Grant No. ts201712098), the National Natural Science Foundation of China (Grant Nos. 82072094 and 82001902).

\section{Availability of data and materials}

The data are available upon request.

\section{Ethics approval and consent to participate}

This study was approved by the Research Ethics Board of the Shandong Cancer Hospital. Written informed consent was waived by the Institutional Review Board.
Consent for publication

Not applicable.

\section{Competing interests}

The authors declare that they have no competing interests.

Received: 10 October 2020 Accepted: 11 February 2021

Published online: 18 February 2021

\section{References}

1. EBCTCG, Darby S, Mcgale P, et al. Effect of radiotherapy after breast-conserving surgery on 10-year recurrence and 15-year breast cancer death: meta-analysis of individual patient data for 10801 women in 17 randomised trials. Lancet. 2011;378(9804):1707-16. https://doi.org/10.1016/ S0140-6736(11)61629-2.

2. Kheirelseid A, Jumustafa $\mathrm{H}$, Miller $\mathrm{N}$, et al. Bilateral breast cancer: analysis of incidence, outcome, survival and disease characteristics. Br Cancer Res Treat. 2011;126(1):131-40. https://doi.org/10.1007/s10549-010-1057-y.

3. Michiels S, Poels K, Crijns W, et al. Volumetric modulated arc therapy of head-and-neck cancer on a fast-rotating o-ring linac: plan quality and delivery time comparison with a c-arm linac. Radiother Oncol. 2018;128(3):479-84. https://doi.org/10.1016/j.radonc.2018.04.021.

4. LiT, Irmen P, Liu H, Shi W, Alonso-Basanta M, Zou W, et al. Dosimetric performance and planning/delivery efficiency of a dual-layer stacked and staggered MLC on treating multiple small targets: a planning study based on single-isocenter multi-target stereotactic radiosurgery (SRS) to brain metastases. Front Oncol. 2019;9:7. https://doi.org/10.3389/ fonc.2019.00007.

5. Barsky AR, O'Grady F, Kennedy C, et al. Initial clinical experience treating patients with breast cancer on a 6-MV flattening-filter-free o-ring linear accelerator. Adv Radiat Oncol. 2019;4(4):571-8. https://doi.org/10.1016/j. adro.2019.05.006.

6. Flores-Martinez E, Kim GY, Yashar CM, Cerviño LI. Dosimetric study of the plan quality and dose to organs at risk on tangential breast treatments using the Halcyon linac. J Appl Clin Med Phys. 2019;20(7):58-67. https:// doi.org/10.1002/acm2.12655.

7. Li C, Chen J, Zhu J, et al. Plan quality comparison for cervical carcinoma treated with Halcyon and Trilogy intensity-modulated radiotherapy. J Cancer. 2019;10(24):6135-41. https://doi.org/10.7150/jca.32500.

8. Seppälä J, Heikkilä J, Myllyoja K, et al. Volumetric modulated arc therapy for synchronous bilateral whole breast irradiation — a case study. Rep Pract Oncol Radiother. 2015;20(5):398-402. https://doi.org/10.1016/j. rpor.2015.05.011.

9. Nicolini G, Clicio A, Fogliata A, et al. Simultaneous integrated boost radiotherapy for bilateral breast: a treatment planning and dosimetric comparison for volumetric modulated arc and fixed field intensity modulated therapy. Radiat Oncol. 2009;24(4):27. https://doi. org/10.1186/1748-717X-4-27.

10. Sun $T$, Lin $X$, Tong $Y$, et al. Heart and cardiac substructure dose sparing in synchronous bilateral breast radiotherapy: a dosimetric study of proton and photon radiation therapy. Front Oncol. 2019;9:1456. https://doi. org/10.3389/fonc.2019.01456.

11. Boman E, Rossi M, Haltamo M, Skyttä T, Kapanen M. A new split arc VMAT technique for lymph node positive breast cancer. Phys Med. 2016;32(16):1428-36. https://doi.org/10.1016/j.ejmp.2016.10.012.

12. Boman $E$, Maija Rossia M, Kapanen $M$. The robustness of dual isocenter VMAT radiation therapy for bilateral lymph node positive breast cancer. Phys Med. 2017;14:11-7. https://doi.org/10.1016/j.ejmp.2017.11.006.

13. Paddick IA. Simple scoring ratio to index the conformity of radiosurgical treatment plans, technical note. J Neurosurg. 2000;93(S3):219-22. https:// doi.org/10.3171/jns.2000.93.supplement_3.0219.

14. Wu Q, Mohan R, Morris M, et al. Simultaneous integrated boost intensitymodulated radiotherapy for locally advanced head-and-neck squamous cell carcinomas. I: dosimetric results. Int J Radiat Oncol Biol Phys. 2003;56(2):573-85. https://doi.org/10.1016/s0360-3016(02)04617-5.

15. Hong TS, Tomé WA, Chappell RJ, et al. The impact of daily setup variations on head-and-neck intensity-modulated radiation therapy. Int J Radiat Oncol Biol Phys. 2005;61(3):779-88. https://doi.org/10.1016/j.jirob p.2004.07.696. 
16. Topolnjak R, Sonke JJ, Nijkamp J, et al. Breast patient setup error assessment: comparison of electronic portal image devices and cone-beam computed tomography matching results. Int J Radiat Oncol Biol Phys. 2010;78(4):1235-43. https://doi.org/10.1016/j.jjrobp.2009.12.021.

17. Bahig H, Roussin É, Yassa M, et al. Partial kilovoltage cone beam computed tomography, complete kilovoltage cone beam computed tomography, and electronic portal images for breast radiation therapy: a dose-comparison study. Pract Radiat Oncol. 2015;5(5):521-9. https://doi. org/10.1016/j.prro.2015.02.009.

18. Rossi M, Boman E, Skyttä T, et al. Dosimetric effects of anatomical deformations and positioning errors in VMAT breast radiotherapy. J Appl Clin Med Phys. 2018;19(5):506-16. https://doi.org/10.1002/acm2.12409.

19. Malajovich I, Teo BK, Petroccia $H$, et al. Characterization of the megavoltage cone-beam computed tomography (MV-CBCT) system on halcyon for IGRT: image quality benchmark, clinical performance, and organ doses. Front Oncol. 2019. https://doi.org/10.3389/fonc.2019.00496.

20. Darby S, Ewertz M, McGale P, et al. Risk of ischemic heart disease in women after radiotherapy for breast cancer. N Engl J Med. 2013;368(11):987-98. https://doi.org/10.1056/NEJMoa1209825.

21. Nilsson $G$, Holmberg $L$, Garmo H, et al. Distribution of coronary artery stenosis after radiation for breast cancer. J Clin Oncol. 2012;30(4):380-6. https://doi.org/10.1200/JCO.2011.34.5900.

22. van den Bogaard VA, Ta BD, van der Schaaf A, et al. Validation and modification of a prediction model for acute cardiac events in patients with breast Cancer treated with radiotherapy based on threedimensional dose distributions to cardiac substructures. J Clin Oncol. 2017:35(11):1171-8. https://doi.org/10.1200/JCO.2016.69.8480.

23. Piroth MD, Baumann R, Budach W, et al. Heart toxicity from breast cancer radiotherapy: current findings, assessment, and prevention. Strahlenther Onkol. 2019;195(1):1-12. https://doi.org/10.1007/s00066-018-1378-z.

24. Fiorentino A, Mazzola R, Naccarato S, et al. Synchronous bilateral breast cancer irradiation: clinical and dosimetrical issues using volumetric modulated arc therapy and simultaneous integrated boost. Radiol Med. 2017;122(6):464-71. https://doi.org/10.1007/s11547-017-0741-y.

25. O'Grady F, Barsky AR, Anamalayil S, et al. Increase in superficial dose in whole-breast irradiation with Halcyon straight-through linac compared with traditional C-arm linac with flattening filter: in vivo dosimetry and planning study. Adv Radiat Oncol. 2019;5(1):120-6. https://doi. org/10.1016/j.adro.2019.07.011.

26. Xiao Y, Kry SF, Popple R, et al. Flattening filter-free accelerators: a report from the AAPM therapy emerging technology assessment work group. J Appl Clin Med Phys. 2015;16(3):5219. https://doi.org/10.1120/jacmp.v16i3 .5219 .

27. Mesbahi A. Dosimetric characteristics of unflattened 6 mv photon beams of a clinical linear accelerator: a Monte Carlo study. Appl Radiat Isot. 2007;65(9):1029-36. https://doi.org/10.1016/j.apradiso.2007.04.023.
28. Schmidhalter D, Malthaner M, Born EJ, et al. Assessment of patient setup errors in IGRT in combination with a six degrees of freedom couch. Z Med Phys. 2014;24(2):307-13. https://doi.org/10.1016/j.zemedi.2013.11.002.

29. Hyde $D$, Lochray $F$, Korol $R$, et al. Spine stereotactic body radiotherapy utilizing cone-beam CT image-guidance with a robotic couch: intrafraction motion analysis accounting for all six degrees of freedom. Int J Radiat Oncol Biol Phys. 2012;82(3):555-62. https://doi.org/10.1016/j.ijrob p.2011.06.1980.

30. Ma Z, Zhang W, Su Y, et al. Optical surface management system for patient positioning in interfractional breast cancer radiotherapy. Biomed Res Int. 2018. https://doi.org/10.1155/2018/6415497.

31. Hamming VC, Visser C, Batin E, et al. Evaluation of a 3D surface imaging system for deep inspiration breath-hold patient positioning and intra-fraction monitoring. Radiat Oncol. 2019;14(1):125. https://doi. org/10.1186/s13014-019-1329-6.

32. Nguyen D, Farah J, Barbet N, Khodri M. Commissioning and performance testing of the first prototype of AlignRT InBore ${ }^{T M}$ a Halcyon ${ }^{T M}$ and Ethos ${ }^{T M}$-dedicated surface guided radiation therapy platform. Phys Med. 2020;80:159-66. https://doi.org/10.1016/j.ejmp.2020.10.024.

33. Bergom C, Currey A, Desai N, Tai A, Strauss JB. Deep inspiration breath hold: techniques and advantages for cardiac sparing during breast cancer irradiation. Front Oncol. 2018;8:87. https://doi.org/10.3389/ fonc.2018.00087

34. Kunheri B, Kotne S, Nair SS, Makuny D. A dosimetric analysis of cardiac dose with or without active breath coordinator moderate deep inspiratory breath hold in left sided breast cancer radiotherapy. J Cancer Res Ther. 2017;13(1):56-61. https://doi.org/10.4103/jcrt.JCRT_1414_16.

35. Mohamad O, Shiao J, Zhao B, Roach K, Ramirez E, Vo D, et al. Deep inspiration breath-hold for left-sided breast cancer patients with unfavorable cardiac anatomy requiring internal mammary nodal irradiation. Pract Radiat Oncol. 2017;7(6):e361-7. https://doi.org/10.1016/j. prro.2017.04.006.

36. Nicolini G, Fogliata A, Clivio A, Vanetti E, Cozzi L. Planning strategies in volumetric modulated arc therapy for breast. Med Phys. 2011;38(7):4025-31. https://doi.org/10.1118/1.3598442.

37. Tyran M, Tallet A, Resbeut M, et al. Safety and benefit of using a virtual bolus during treatment planning for breast cancer treated with arc therapy. J Appl Clin Med Phys. 2018;19(5):463-72. https://doi.org/10.1002/ acm2.12398.

\section{Publisher's Note}

Springer Nature remains neutral with regard to jurisdictional claims in published maps and institutional affiliations.
Ready to submit your research? Choose BMC and benefit from:

- fast, convenient online submission

- thorough peer review by experienced researchers in your field

- rapid publication on acceptance

- support for research data, including large and complex data types

- gold Open Access which fosters wider collaboration and increased citations

- maximum visibility for your research: over $100 \mathrm{M}$ website views per year

At BMC, research is always in progress.

Learn more biomedcentral.com/submissions 